\title{
Acute transverse myelitis in a HIV-positive patient with COVID-19
}

\author{
${ }^{* 1}$ Victor Nemtan, ${ }^{1}$ Evghenia Hacina, ${ }^{1}$ Galina Topciu, ${ }^{2}$ Elena Manole, ${ }^{2}$ Vitalie Lisnic, \\ ${ }^{3}$ Majda Thurnher, ${ }^{4}$ Rüdiger von Kummer \\ ${ }^{1}$ Medical Department of Security and Intelligence Service of the Republic of Moldova \\ Chisinau, the Republic of Moldova \\ ${ }^{2}$ Department of Neurology No 1, Nicolae Testemitanu State University of Medicine and Pharmacy \\ Chisinau, the Republic of Moldova \\ ${ }^{3}$ Department of Biomedical Imaging and Image-Guided Therapy, Medical University Vienna, Austria \\ ${ }^{4}$ Institute of Diagnostic and Interventional Neuroradiology, Universitätsklinikum Dresden, Germany \\ Authors' ORCID iDs, academic degrees and contributions are available at the end of the article \\ ${ }^{\star}$ Corresponding author: victor.nemtsan@gmail.com \\ Manuscript received August 31, 2020; revised manuscript September 24, 2020; published online October 02, 2020
}

\begin{abstract}
Background: Immunocompromised status keeps on being a challenge for a physician, especially in the context of the coronavirus disease - 19 (COVID-19) pandemic. The predominant clinical presentations are related to the respiratory system, but neurological manifestations are recognized increasingly. Cases of myelitis associated with the new coronavirus infection have already been published, but no cases of HIV-positive patients with myelitis and COVID-19 have been reported yet.

Material and methods: This study described a clinical case of a human-immunodeficiency virus (HIV) - positive patient, who developed an acute transverse myelitis with confirmed SARS-CoV-2 infection.

Results: Magnetic Resonance Imaging examination showed longitudinally extensive spinal cord abnormality, and laboratory tests confirmed SARS-CoV-2 infection. The patient responded to methylprednisolone pulse therapy, followed by oral corticosteroids and therapeutic plasma exchange.

Conclusions: Continuing pandemic and the expectation that a large part of the world population will be infected suggest that the number of patients with neurological manifestations could become large. Curious neurologic constellations can appear which complicate the diagnostic process and treatment in certain patients.

Key words: COVID-19, transverse myelitis, neuromyelitis optica spectrum disorder.
\end{abstract}

Cite this article

Nemtan V, Hacina E, Topciu G, Manole E, Lisnic V, Thurnher M, von Kummer R. Acute transverse myelirtis in a HIV-positive patient with COVID-19. Mold Med J. 2020;63(5):51-53. doi: 10.5281/zenodo.4018955.

\section{Introduction}

By September 1, 2020 the coronavirus disease - 19 (COVID-19) pandemic has resulted in more than 25 million confirmed cases and more than 850000 deaths [1]. The predominant clinical presentations are related to the respiratory system; however, neurological manifestations are recognized increasingly. These features are determined by the effects of a combination of direct viral infection, inflammation of the structures of the nervous system and vasculature, nonspecific complications of systemic disease, which can be para-infectious or post-infectious [2].

Transverse myelitis (TM) is a focal disorder of the spinal cord presenting in acute or subacute manner (hours/days) resulting in motor, sensory, and autonomic dysfunction of varying degrees of expression depending on the type, location and surface of the lesion longitudinally and transversely. The term longitudinally extensive transverse myelitis is used when the spinal cord lesion extends over three or more vertebral segments.
The possible causes of myelitis are as follows: infections (viruses, bacteria, fungi, and parasites), post-infectious or post-vaccination, paraneoplastic, demyelinating (multiple sclerosis (MS), neuromyelitis optica (NMO), acute disseminated encephalomyelitis, other inflammatory disorders (neurosarcoidosis, systemic lupus erythematosus, Sjögren syndrome, Behcet's disease), congestive edema due to dural arteriovenous fistula, and tumor $[3,4]$. TM may be idiopathic in $15-30 \%$ of cases. Post-infectious and idiopathic myelitis incidence varies between 1.3 - 8 cases / 1 million, but can reach 24.6 cases / million in patients with MS [5]. Recent case reports of myelitis associated with the new coronavirus infection were published, but the mechanism of spinal cord injury remains unclear $[6,7]$.

Human-immunodeficiency virus (HIV) causes spinal cord injury both by direct HIV virus invasion, presenting in vacuolar myelitis in most cases, as well as manifestations of opportunistic virus-induced infections (Herpes simplex virus (HSV), Cytomegalovirus (CMV), Epstein-Barr virus (EBV), Varicella Zoster virus, or bacteria (Mycobacterium 
tuberculosis, Treponema pallidum), as well as due to vitamin B12 deficiency. Vacuolar myelopathy (VM) is symptomatic in $5-10 \%$ of acquired immune deficiency syndrome (AIDS) patients, shows a progressive evolution and is pathologically characterized by the presence of intralamellar white matter vacuoles in the posterior and lateral columns of the thoracic spinal cord [8].

SARS-CoV-2 causing the COVID-19 pandemic is proven to be neurotropic and may affect the central and peripheral nervous system. The mechanism of the new type of coronavirus neuroinvasion has not yet been fully identified $[2,9]$. Short- and long-term health effects for patients who have been infected have not yet been assessed. The changes induced by the new type of coronavirus in the coagulation status resulted in an impressive number of fatal thromboembolic events, including the cerebral ones. Guillain-Barré syndrome reported in COVID-positive patients is explained as a possible parainfectious manifestation, whereas cases of necrotizing hemorrhagic meningoencephalitis, altered mental status, and respiratory distress syndrome are referred to both direct virus action on brain neurons, as well as cytokine storm syndrome $[10,11]$.

\section{Material and methods}

The present study reported a clinical case of a humanimmunodeficiency virus (HIV) - positive patient who developed an acute TM with confirmed SARS-CoV-2 infection.

\section{Case report}

A 27-year-old male, with known HIV infection for the past 1 year, treated with anti-retroviral therapy, was hospitalized on June 7, 2020 due to paresthesia and numbness in legs and in the right arm, paralysis in lower extremities, and bladder and bowel dysfunction (retention). All these symptoms developed quickly, reaching the nadir in 15 hours.

Clinical evaluation revealed normal cranial nerves function, spastic tetraparesis, with 4/4.5 Medical Research Council (MRC) in upper and 0.5/2MRC in lower extremities, Th7 superficial and C7 deep sensory level disturbances. The patient was subfebrile $37.5 \mathrm{C}$ without other systemic abnormalities.

An extensive paraclinical workup was obtained. Routine laboratory data showed a slight general blood inflammatory abnormalities (WBC $13.7 \times 10^{9}$, ESR $14 \mathrm{~mm} / \mathrm{h}$ ) but with negative CRP. His CD4 count at admission was 310 cells/ $\mu \mathrm{l}$, and viral load was less than 40 copies $/ \mathrm{ml}$. Cerebrospinal fluid (CSF) analysis was normal. Blood serology and CSF polymerase chain reaction (PCR) for HSV 1,2,6, CMV, EBV, Borrelia burgdorferi, Treponema pallidum, Toxoplasma gondii, Chlamydia trachomatis, Mycoplasma pneumoniae, Ureaplasma urealyticum were negative. Autoimmune markers tests (autoantibodies ANA, ANCA), tumor marker tests (CA 19.9, Ca 15.3, PSA, CEA, Alpha Fetoprotein) and hepatitis viral serology all were negative. Thyroid function tests were normal. The first swab for SARS-CoV-2 at admission was negative. CSF oligoclonal bands (OCB) and serum anti-aquaporin-4 antibody (AQP4-IgG), anti-MOG anti- bodies were negative. Vitamin B12, methylmalonic acid and angiotensin-converting enzyme levels were normal. Folic acid level test was $4.62 \mathrm{nmol} / \mathrm{L}$ (normal range 6.00-39.0). Brain MRI did not show any abnormalities. Spinal cord MRI revealed an extensive C4-Th5 lesion mainly in posterior columns and right lateral column without gadolinium enhancement (fig. 1).

The patient was treated with IV methylprednisolone $1 \mathrm{~g} /$ day for 5 days, followed by oral corticosteroids and five procedures of plasma exchanges. His condition improved with a significant reduction in paresis. Lung CT performed on the $19^{\text {th }}$ day of illness showed slight patchy ground-glass opacity basal on the left side. No other typical symptoms for CoV-2 infection were noted. A repeated swab for SARSCoV-2 was positive, followed by negative COVID-19 PCR in the CSF. The 1-month follow-up spinal MRI did not reveal significant changes.

Two-month follow-up revealed significant improvement of muscle strength allowing the patient to walk. Sphincter disturbances became less severe. At the same time, the CD4 count dropped to less than 200 cells/ $\mu$ l.

\section{Discussion}

The presented case is unique for several reasons. All these circumstances - transverse myelitis, HIV infection and COVID-19 may be a random combination, as well as a range of three successive elements or an interrelationship that influenced the onset of clinical signs and imaging changes. Normal CSF examination does not meet the TM diagnostic criteria [12]. Negative data for OCB and AQP4IgG, and normal brain imaging disclaim the most common demyelinating causes of TM-MS and NMO, but do not rule out a possible isolated clinical syndrome or NMO spectrum disorders.

Spinal cord injury presented in posterior and lateral columns suggested a subacute combined degeneration due to vitamin B12 deficiency. Neurosyphilis and neurosarcoidosis were excluded by laboratory and imaging studies.

Despite the patient's CD4 cells count that did not indicate a severe degree of immunosuppression, the risk of opportunistic infections as a cause of myelitis had to be considered. However, the absence of CSF pleocytosis and normal infectious disease tests did not confirm this hypothesis. HIV-associated spinal cord disease cannot be excluded, particularly vacuolar myelitis, which is slowly progressive and that predominantly occur at AIDS stage. The clinical examination usually demonstrates motor and sphincter disorders, sensitive ataxia, though without a sensory level, as well as normal or discreetly modified MRI that often develops a simultaneous cognitive impairment [8].

The patient's SARS-CoV-2 infection role is controversial. This could be an accidental co-infection during hospitalization, a fact supported by the negative test results at admission and the lack of typical blood changes. At the same time, a false-negative result cannot be denied. High-dose corticosteroids therapy and plasma exchange might influence 

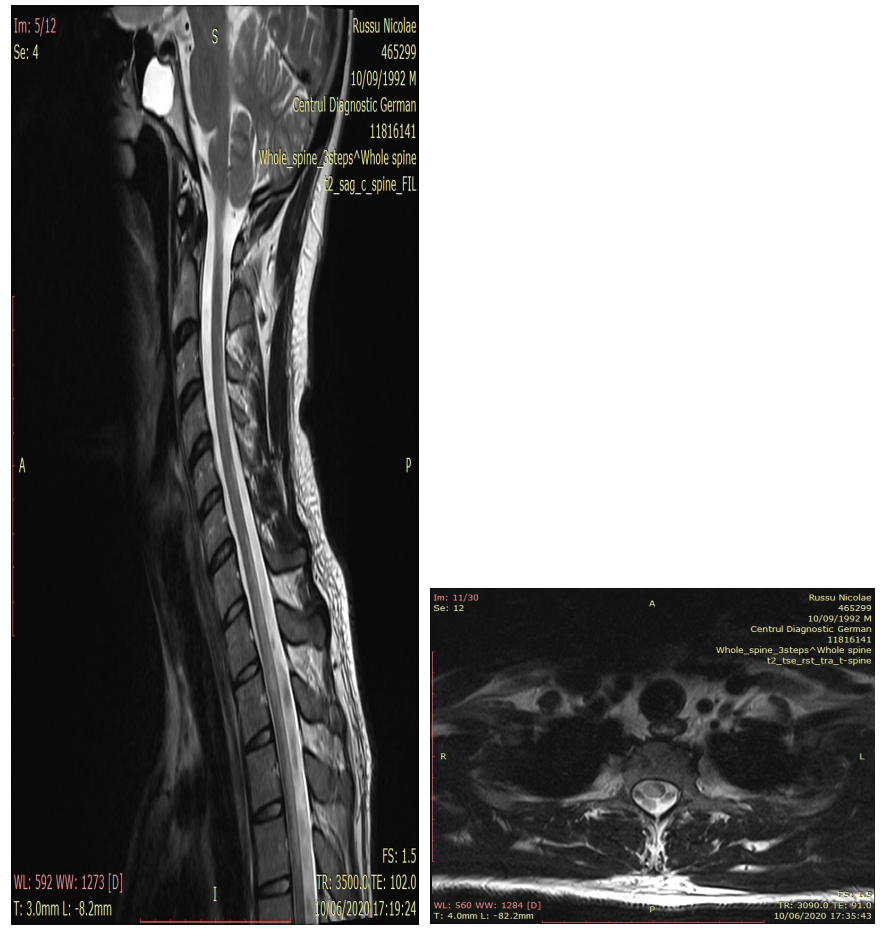

Fig. 1. Sagittal and axial MRI shows the spinal cord segmental myelopathy area at $\mathrm{C} 4-\mathrm{T} 5$ level, $\sim 15 \mathrm{~cm}$ in length, with a predominant involvement of the posterior and right lateral columns, without contrast uptake

the relatively benign course of SARS-CoV-2 pneumonia. Myelitis may resemble a direct virus injury, or a parainfectious autoimmune process, as well due to molecular mimicry, the fact sustained by the positive response to corticosteroid therapy.

\section{Conclusions}

Continuing pandemic and the expectation that a large part of the world population will be infected, suggest that the number of patients with neurological manifestations could become greater. Curious neurologic constellations can appear which complicate the diagnostic process and treatment in certain patients.

\section{References}

1. Worldometers.info. COVID-19 coronavirus pandemic. Dover, Delaware, USA: Worldometers.info; 2020 [cited 2020 Jul 21]. Available from: https://www.worldometers.info/coronavirus/

2. Ellul MA, Benjamin L, Singh B, et al. Neurological associations of COVID-19. Lancet Neurol. 2020;(19):767-83. doi: 10.1016/S14744422(20)30221-0.

3. Jacob A, Weinshenker B. An approach to the diagnosis of acute transverse myelitis. Semin Neurol. 2008 Feb;28(1):105-120. doi: 10.1055/s-20071019132.

4. Murphy OC, Salazar-Camelo A, Jimenez JA, et al. Clinical and MRI phenotypes of sarcoidosis-associated myelopathy. Neurol Neuroimmunol Neuroinflamm. 2020;7(4):e722. doi: 10.1212/NXI.0000000000000722.

5. Frohman EM, Wingerchuk DM. Transverse myelitis. N Engl J Med. 2010;363:564-72. doi: 10.1056/NEJMcp1001112.

6. Sotoca H, Rodriguez-Alvarez Y. COVID-19-associated acute necrotizing myelitis. Neurol Neuroimmunol Neuroinflamm. 2020;7(5):e803. doi: 10.1212/NXI.0000000000000803.

7. AlKetbi R, AlNuaimi D, AlMulla M. et al. Acute myelitis as a neurological complication of Covid-19: a case report and MRI findings. Radiol Case Rep. 2020;15(9):1591-1595. doi: 10.1016/j.radcr.2020.06.001.

8. McArthur JK, Brew B, Nath A. Neurological complication of HIV infection. Lancet Neurol. 2005;4(9):543-55. doi: 10.1016/S14744422(05)70165-4.

9. Netland J, Meyerholz DK, Moore S, et al. Severe acute respiratory syndrome coronavirus infection causes neuronal death in the absence of encephalitis in mice transgenic for human $\mathrm{ACE}_{2}$. J Virol. 2008 Aug;82(15):7264-75. doi: 10.1128/JVI.00737-08.

10. Beghi E, Feigin V, Caso V, et al. COVID-19 infection and neurological complications: present findings and future predictions. Neuroepidemiology. 2020. Published online: July 1, 2020. doi: 10.1159/000508991.

11. Roman G, Spencer PS, Reis J, et al. The neurology of COVID-19 revisited: A proposal from the Environmental Neurology Specialty Group of the World Federation of Neurology to implement international neurological registries. J Neurol Sci. 2020 Jul;414(15):116884. doi: 10.1016/j. jns.2020.116884.

12. Transverse Myelitis Consortium Working Group. Proposed diagnostic criteria and nosology of acute transverse myelitis. Neurology. 2002;59(4):499-505. doi: 10.1212/wnl.59.4.499.

\section{Authors' ORCID iDs and academic degrees}

Victor Nemtan, MD, PhD - https://orcid.org/0000-0002-5247-7316.

Evghenia Hacina, MD - https://orcid.org/0000-0002-5785-6710.

Galina Topciu, MD - https://orcid.org/0000-0001-8991-4586.

Elena Manole, MD, PhD, Associate Professor - https://orcid.org/0000-0003-0164-859X.

Vitalie Lisnic, MD, PhD, Professor - https://orcid.org/0000-0002-5432-8859.

Majda Thurnher, MD, PhD, Professor - https://orcid.org/0000-0001-9746-0932.

Rudiger von Kummer, MD, PhD, Professor - https://orcid.org/0000-0003-0119-4604.

\section{Authors' contribution}

VN, EH, GT, EM acquired, interpreted the data, drafted the first manuscript. VL designed the study. MT, RC interpreted the radiological data, guided the diagnostic pathway. All the authors revised and approved the final version of the manuscript.

\section{Funding}

The study was supported by Nicolae Testemitanu State University of Medicine and Pharmacy.

The authors are independent and take responsibility for the integrity of the data and accuracy of the data analysis.

\section{Ethical approval}

The patient consented to publish his anonymized health data.

\section{Conflict of interests}

The authors declare that they have no conflict of interests. 